\title{
Simultaneous SNP identification and assessment of allele-specific bias from ChIP-seq data
}

\author{
Yunyun $\mathrm{Ni}$, Amelia Weber Hall, Anna Battenhouse and Vishwanath R lyer
}

\begin{abstract}
Background: Single nucleotide polymorphisms (SNPs) have been associated with many aspects of human development and disease, and many non-coding SNPs associated with disease risk are presumed to affect gene regulation. We have previously shown that SNPs within transcription factor binding sites can affect transcription factor binding in an allele-specific and heritable manner. However, such analysis has relied on prior whole-genome genotypes provided by large external projects such as HapMap and the 1000 Genomes Project. This requirement limits the study of allele-specific effects of SNPs in primary patient samples from diseases of interest, where complete genotypes are not readily available.

Results: In this study, we show that we are able to identify SNPs de novo and accurately from ChIP-seq data generated in the ENCODE Project. Our de novo identified SNPs from ChIP-seq data are highly concordant with published genotypes. Independent experimental verification of more than 100 sites estimates our false discovery rate at less than 5\%. Analysis of transcription factor binding at de novo identified SNPs revealed widespread heritable allele-specific binding, confirming previous observations. SNPs identified from ChIP-seq datasets were significantly enriched for disease-associated variants, and we identified dozens of allele-specific binding events in non-coding regions that could distinguish between disease and normal haplotypes.

Conclusions: Our approach combines SNP discovery, genotyping and allele-specific analysis, but is selectively focused on functional regulatory elements occupied by transcription factors or epigenetic marks, and will therefore be valuable for identifying the functional regulatory consequences of non-coding SNPs in primary disease samples.
\end{abstract}

Keywords: SNPs, Transcription factors, ChIP-seq, Genotyping, Allele-specific

\section{Background}

Single nucleotide polymorphisms (SNPs) have been associated with normal variation in biological traits as well as many human diseases [1,2]. With recent advances in genotyping and sequencing technologies, the number of known SNPs has increased dramatically. Several international collaborative projects such as the HapMap project [3] and the 1000 Genomes Project [4] have genotyped millions of SNPs in hundreds of human individuals. In the dbSNP 129 build (http://www.ncbi.nlm.nih. gov/projects/SNP/), approximately 52 million SNPs are cataloged in humans. However, despite the availability of these massive datasets, our understanding of the functional importance of most genomic variants is still very

\footnotetext{
*Correspondence: vishy.iyer@gmail.com

Center for Systems and Synthetic Biology, Institute for Cellular and Molecular Biology, Section of Molecular Genetics and Microbiology, University of Texas at Austin, Austin, TX 78712, USA
}

limited. Genome-wide association (GWA) studies have been the main method to associate genomic variants with a variety of phenotypes including diseases. SNP genotyping is performed in large numbers of disease individuals and controls to find statistically significant associations between SNPs and diseases [5]. So far, GWA studies have identified approximately 7000 SNPs or regions as disease associated [1], although in most cases neither the causal SNPs nor the molecular mechanisms are known (http://www.genome.gov/gwastudies/).

Although SNPs located within coding regions that affect protein sequences are the most obvious candidates for functional SNPs, among the 7000 disease associated SNPs, only about $7 \%$ affect coding regions. For the majority of SNPs occurring in non-coding regions and shown to be associated with disease risk, there are two possibilities for explaining this association. First the identified or imputed SNPs could directly contribute to disease risk. Alternatively,

\section{Biomed Central}

(c) 2012 Ni et al. licensee BioMed Central Ltd. This is an Open Access article distributed under the terms of the Creative Commons Attribution License (http://creativecommons.org/licenses/by/2.0), which permits unrestricted use, distribution, and reproduction in any medium, provided the original work is properly cited. 
it has been proposed that identified SNPs could be in linkage disequilibrium (LD) with rarer and unknown causative SNPs that are not directly assayed or imputed [6-8]. In either case, it raises the question of how common or rare variants in non-coding regions could contribute to disease risk. One explanation for the functional effects of noncoding SNPs in introns and intergenic regions is that they affect transcription regulation, splicing or other aspects of RNA processing or stability $[9,10]$. For example, a SNP upstream of MUC5B has been shown to regulate its expression and is associated with familial interstitial pneumonia and idiopathic pulmonary fibrosis [11]. Diseaseassociated SNPs have also been reported to directly affect transcription factor binding. Two non-coding regulatory SNPs in FGFR2 affect binding of the transcription factors Oct-1/Runx2 and C/EBP $\beta$, leading to an increased expression of FGFR2 in the rarer homozygous genotypes which have increased breast cancer risk [12]. A SNP upstream of MYC has been shown to affect the binding of transcription factor YY1 which may serve to regulate MYC expression in prostate cancers [13]. Two SNPs in the intronic promoter of MDM2, an oncogene that downregulates the tumor suppressor TP53, have independent and opposite effects on the binding of the transcription factor SP1. However, the combination of both SNPs into a commonly observed haplotype reduces SP1 binding in the MDM2 promoter and reduces breast and ovarian cancer risk, likely by reducing MDM2 expression [14,15]. While these studies shed light on the functional basis of regulatory SNPs, they account for a very small fraction of known disease associated non-coding SNPs.

We have previously shown that the transcription factor CTCF exhibits allele-specific binding bias at hundreds of heterozygous SNP sites in the human genome [16]. This study and others [17] showed that not only is there widespread allele-specific binding of transcription factors, but suggested a genetic basis for this specificity, with nucleotide polymorphisms directly affecting the binding of transcription factors. In principle therefore, it should be possible to examine transcription factor binding and other features of gene expression such as chromatin modifications and RNA expression levels in disease cells to identify whether polymorphisms associated with disease risk indeed affect transcription factor binding, chromatin, and/or RNA in an allele-specific manner. However, most previous analyses of allelespecific transcription factor binding and chromatin depended upon the availability of individual genotypes from the 1000 Genomes Project, and therefore cannot be easily performed in primary patient samples, where whole-genome genotyping data based on deep sequencing is generally not available. We have now developed an approach for simultaneous SNP discovery and transcription factor allele-specific binding analysis from
ChIP-seq data we have generated in the ENCODE Project, without reference to preexisting genotyping information. This approach has enabled us to examine allele specific transcription factor binding in any cell where ChIP-seq data is available and paves the way for extending such analysis of other sequencing-based readouts of gene regulatory mechanisms to primary cell samples from individuals without pre-existing genotype data.

\section{Results}

\section{SNP discovery from ChIP-seq data}

We carried out SNP discovery from ChIP-seq data that we generated for the transcription factor CTCF in 10 human cell lines, including six lymphoblastoid cell lines that had been previously sequenced and genotyped by the 1000 Genomes Project, embryonic stem cells (H1 ESCs), vascular endothelial cells (HUVEC), and normal and disease fibroblasts (Table 1) $[16,18]$. In addition we carried out SNP discovery from RNA pol II (RNAPII) and H3K4me3 ChIP-seq data from GM12878 cells, an ENCODE Tier 1 cell line. Besides the above call sets which we analyzed in detail, we also called SNPs in H3K4me3 and/or H3K27me3 ChIP-seq data generated by ENCODE from 17 other cell lines as well as from RNA-seq data in GM12891 to demonstrate that the pipeline is broadly applicable (Methods). In order to identify SNPs, we adapted the variant discovery software suite developed by the 1000 Genomes Project, the Genome Analysis ToolKit (GATK) $[19,20]$. Raw Illumina sequences were aligned to

\section{Table 1 Statistics of SNP discovery}

\begin{tabular}{llccccc}
\hline Cell line & factor & $\begin{array}{c}\text { aligned } \\
\text { reads }\end{array}$ & $\begin{array}{c}\text { Ht5 } \\
\text { regions }\end{array}$ & $\begin{array}{c}\text { total } \\
\text { SNPs }\end{array}$ & $\begin{array}{c}\text { novel } \\
\text { SNPs }\end{array}$ & Ti/Tv \\
\hline GM12878 & CTCF & $30,400,254$ & $0.40 \%$ & 112,999 & $2989(2.6 \%)$ & 2.04 \\
GM12891 & CTCF & $28,282,066$ & $0.45 \%$ & 137,056 & $3443(2.5 \%)$ & 2.18 \\
GM12892 & CTCF & $41,857,998$ & $0.75 \%$ & 206,349 & $5439(2.6 \%)$ & 2.13 \\
GM19238 & CTCF & $31,125,372$ & $0.53 \%$ & 154,211 & $7474(4.8 \%)$ & 2.11 \\
GM19239 & CTCF & $24,857,361$ & $0.41 \%$ & 153,092 & $7034(4.6 \%)$ & 2.13 \\
GM19240 & CTCF & $32,009,059$ & $0.45 \%$ & 144,088 & $7832(5.4 \%)$ & 2.11 \\
GM12878 & RNAPII & $85,763,827$ & $0.55 \%$ & 152,071 & $5720(3.8 \%)$ & 2.03 \\
GM12878 & H3K4me3 & $74,464,458$ & $1.95 \%$ & 200,675 & $8231(4.1 \%)$ & 1.99 \\
FB8470 & CTCF & $39,394,358$ & $0.47 \%$ & 173,336 & $4344(2.5 \%)$ & 2.11 \\
H1 ESC & CTCF & $14,462,504$ & $0.28 \%$ & 94,398 & $2308(2.4 \%)$ & 2.18 \\
Progeria & CTCF & $46,925,953$ & $0.47 \%$ & 220,871 & $5328(2.4 \%)$ & 2.06 \\
HUVEC & CTCF & $21,734,605$ & $0.32 \%$ & 55,492 & $1117(2.0 \%)$ & 2.08 \\
\hline
\end{tabular}

GM lines are lymphoblastoid cells resequenced by the 1000 Genomes Project. FB8470 is a normal fibroblast line and Progeria refers to a fibroblast line obtained from patients with the disease. H1 ESCs are human embryonic stem cells and HUVECs are human vascular endothelial cells. $\mathrm{Ht} 5$ regions are regions covered by at least 5 reads in ChIP-seq data. The fraction of the genome represented in $\mathrm{Ht} 5$ regions is shown. Novel SNPs are with reference to dbSNP129. Ti/Tv is the transition to transversion ratio. 
the NCBI36 (hg18) reference human genome sequence with BWA [21] and the alignment was first processed to minimize the effect of duplicated reads, sequencing and alignment errors on genotype calls. The cleaned up alignments were then used to calculate genotype likelihoods and initial SNP calls were made (Methods, Additional file 1: Figure S1). Because of our interest in examining how polymorphisms affect the binding of a sequence-specific transcription factor, we focused exclusively on single nucleotide substitutions and did not consider small insertions/deletions (indels) or larger structural variants in our analysis. While small indels were readily observed in the ChIP-seq alignments (see below), larger structural variants would be missed given the nature of the ChIP-seq data. We filtered the initial SNP call set using two methods, model based filtering and hard filtering. We carried out model-based filtering as implemented in GATK. Briefly, a Gaussian mixture model was built with those initially called SNPs that overlapped with known high-quality SNPs identified by the HapMap and the 1000 Genomes Projects, using their specified annotations. Then all called SNPs were fitted into the model and thresholds were set so that $99 \%$ of the known high-quality SNPs were retained $[19,20]$. Hard filtering was a heuristic approach in which we excluded SNPs from the initial called set that met the following criteria: more than 2 SNPs within 10 base pairs, more than $10 \%$ of the sequence reads overlapping the SNP were not uniquely aligned, SNPs with quality scores less than 50, SNPs overlapping with repeat regions [22], SNPs within 5 bp of indels and SNPs located within regions with exceptionally high read coverage [23]. We considered only SNPs that passed both the model filter and the hard filter for all subsequent analysis presented here.

Table 1 shows a summary of the identified SNPs. Among all CTCF ChIP samples, the total number of SNPs discovered was proportional to the number of aligned reads, with an average of about 150,000 SNPs per individual. Since different transcription factors have different binding properties, the same number of reads may lead to different genome coverage. For example, RNAPII ChIP-seq in GM12878 had more aligned reads than H3K4me3 ChIPseq, but the fraction of the genome covered by more than 5 reads in RNAPII ChIP-seq (0.55\%) was less than that in H3K4me3 ChIP-seq. As a result, more SNPs were discovered in H3K4me3 ChIP-seq than in RNAPII. The transition to transversion ratio $(\mathrm{Ti} / \mathrm{Tv}$ ) for de novo identified SNPs was around 2.1, close to what is estimated in mammals [19,24].

\section{SNPs discovered from ChIP-seq overlap well with 1000 genomes project SNPs}

In the pilot phase of the 1000 Genomes Project, two trios with both parents and a child (CEU and YRI) were sequenced to an average depth of $42 \mathrm{X}$ coverage, and complete genotype information for these six individuals was published [4]. To test whether the SNPs we discovered from our ChIP-seq data from these samples were in agreement with the published genotypes, we performed overlap analysis between SNPs discovered from CTCF ChIP-seq and those published by the 1000 Genomes Project (Pilot 2 SNPs, July, 2010 release).

More than $90 \%$ of the SNPs we identified de novo using CTCF ChIP-seq data in the previously resequenced cell lines overlapped Pilot 2 SNP calls. Because DNA recovered after ChIP represents only a small portion of the genome, we discovered only $3-7 \%$ of all Pilot 2 SNPs. However, if we considered only Pilot 2 SNPs within regions where we had at least $5 \mathrm{X}$ coverage in the CTCF ChIP, more than $70 \%$ of such Pilot 2 SNPs were recovered in our discovery (Table 2). Furthermore, the percent recovery of Pilot 2 SNPs in ChIP-ed regions correlated well with the proportion of the genome covered in ChIPed regions (Additional file 1: Figure S2). SNPs we discovered from ChIPseq datasets for RNAPII and H3K4me3 in GM12878 also showed the same high overlap with Pilot 2 SNPs in this cell line (Table 2).

To independently verify SNPs found in ChIP-seq data, we carried out Sanger sequencing of genomic DNA at several randomly selected loci. We examined a total of 133 sites across 8 cell lines. All tested sites except for six were confirmed to be correctly identified SNPs, suggesting a $5 \%$ error rate for our ChIP-seq genotypes (Table 2, Additional file 1: Figure S3). However, 2 of these 6 discrepancies were cases where the genotype called by ChIP-seq matched the 1000 Genomes genotype, and in the remaining cases, either the alleles identified by ChIPseq matched the alleles known to occur at that location, or the discrepancy could be explained by low sequencing coverage by ChIP-seq, suggesting that our error rate is in fact lower (Additional file 1: Table S1).

The overlap between ChIP and Pilot 2 SNPs described above consider a SNP as overlapping between the two sets when their location and the called alt allele was the same. If we required an exact genotype match between the two sets, the percent overlap was somewhat lower (Additional file 1: Figure S4A). ChIP SNPs had a lower heterozygosity rate than Pilot 2 SNPs, suggesting that some heterozygous SNPs had insufficient read coverage and therefore were called homozygous (Additional file 1: Figure S4B). If we filtered out SNPs with low read coverage, the heterozygosity rate of ChIP SNPs was similar to that of Pilot 2 SNPs, and the exact genotype overlap between ChIP SNPs and Pilot 2 SNPs was also restored to about 90\% (Additional file 1: Figure S4C). We were also able to identify small indels from ChIP-seq data and the majority of these indels also overlapped with those called by the 1000 Genomes Project (Additional file 1: Table S2). 
Table 2 Quality assessment of the discovered SNPs. Pilot 2 SNPs are identified by the 1000 Genomes Project

\begin{tabular}{|c|c|c|c|c|c|c|c|c|}
\hline cell line & factor & $\begin{array}{l}\text { total } \\
\text { SNPs }\end{array}$ & $\begin{array}{c}\text { overlap } \\
\text { with Pilot } 2\end{array}$ & $\begin{array}{l}\text { Pilot } 2 \text { SNPs } \\
\text { recovered } \\
\text { by ChIP }\end{array}$ & $\begin{array}{l}\text { Pilot } 2 \text { SNPs in } \\
\mathrm{Ht} 5 \text { region }\end{array}$ & $\begin{array}{l}\text { Pilot } 2 \text { SNPs in } \\
\text { Ht5 regions } \\
\text { recovered by ChIP }\end{array}$ & $\begin{array}{l}\text { validated/tested } \\
\text { G1000 SNPs }\end{array}$ & $\begin{array}{l}\text { Validated/Tested } \\
\text { novel SNPs }\end{array}$ \\
\hline GM12878 & CTCF & 112,999 & $94.14 \%$ & $3.85 \%$ & 11,908 & $75.37 \%$ & $15 / 17$ & $4 / 5$ \\
\hline GM12891 & CTCF & 137,056 & $90.39 \%$ & $4.55 \%$ & 12,837 & $74.12 \%$ & $13 / 13$ & $4 / 4$ \\
\hline GM12892 & CTCF & 206,349 & $92.23 \%$ & $6.95 \%$ & 20,772 & $76.54 \%$ & $13 / 13$ & $7 / 7$ \\
\hline GM19238 & CTCF & 154,211 & $92.25 \%$ & $4.47 \%$ & 18,863 & $74.50 \%$ & $11 / 11$ & $3 / 3$ \\
\hline GM19239 & CTCF & 153,092 & $94.33 \%$ & $4.43 \%$ & 14,844 & $70.37 \%$ & $15 / 15$ & $2 / 2$ \\
\hline GM19240 & CTCF & 144,088 & $94.48 \%$ & $4.08 \%$ & 17,143 & $74.09 \%$ & $10 / 10$ & $3 / 3$ \\
\hline GM12878 & RNAP\|I & 152,071 & $93.45 \%$ & $5.14 \%$ & 8,368 & $77.84 \%$ & $5 / 6$ & $2 / 2$ \\
\hline GM12878 & H3K4me3 & 200,675 & $93.19 \%$ & $6.76 \%$ & 44,755 & $79.40 \%$ & $1 / 1$ & $1 / 1$ \\
\hline FB8470 & CTCF & 173,336 & N/A & N/A & $\mathrm{N} / \mathrm{A}$ & N/A & N/A & $0 / 0$ \\
\hline H1 ESC & CTCF & 94,398 & N/A & N/A & $\mathrm{N} / \mathrm{A}$ & N/A & N/A & $0 / 0$ \\
\hline Progeria & CTCF & 220,871 & N/A & N/A & N/A & N/A & N/A & $7 / 7$ \\
\hline HUVEC & CTCF & 55,492 & N/A & N/A & N/A & N/A & N/A & $7 / 7$ \\
\hline Progeria & RNAPII & 150,157 & N/A & N/A & N/A & N/A & N/A & $5 / 7$ \\
\hline
\end{tabular}

G1000 SNPs are ChIP-seq discovered SNPs that overlap with Pilot 2 SNPs. N/A is not applicable, as these cells were not genotyped by the 1000 Genomes Project.

SNPs overlapping with 1000 genomes project and novel SNPs are qualitatively similar

In order to characterize in more detail the SNPs we discovered de novo from ChIP-seq data, we separated them into SNPs that overlapped with those found by the 1000 Genomes Project Pilot 2 dataset (referred to here as G1000 SNPs) and those that were not found in Pilot 2 and were therefore novel (novel SNPs). First, we established by genomic sequencing that our novel SNPs were as accurate as G1000 SNPs (Table 2, Additional file 1: Figure S3). We next investigated whether G1000 SNPs were different from novel SNPs in terms of their quality metrics. SNP quality scores reported by the GATK pipeline are Phred-scaled probability scores of the existence of a polymorphism. We found that the median quality scores of novel SNPs were equal to or greater than the mean quality scores of G1000 SNPs (Figure 1A). We then compared the genotype scores which represent the confidence of the genotype called between G1000 SNPs and novel SNPs, and found that novel SNPs had equal or higher genotype scores than G1000 SNPs (Figure 1B). We also examined the distribution of SNPs among six individuals. If novel SNPs were largely erroneous, they might be expected to be found in a single individual but not shared across multiple individuals. However, more than half of the novel SNPs we found were observed in at least 3 individuals and the overall distribution across individuals was also indistinguishable between G1000 and novel SNPs (Figure 1 C, Additional file 1: Figure S5). Finally, we checked our novel SNPs against the larger set of SNP calls made by the 1000 Genomes project at low coverage across a large number of individuals from the same populations. $71 \%$ - $86 \%$ of the SNPs that were novel in a given cell type were indeed found in other individuals in the low coverage set, suggesting that they were not spurious (Additional file 1: Table S3).

\section{Genomic and chromosomal distribution of discovered SNPs}

We examined the genomic distribution of the SNPs we identified from ChIP-seq data. Compared with Pilot 2 SNPs, a larger proportion of the SNPs we discovered from RNAPII and H3K4me3 ChIP-seq data were localized to the 5' UTR, reflecting RNAPII and H3K4me3 localization over the transcription start site (TSS) region (Figure 2A). In contrast, SNPs from CTCF ChIP-seq data showed a genomic location profile similar to Pilot 2 SNPs, both in the GM12878 lymphoblastoid cell line and in a Progeria fibroblast line (Figure 2A), reflecting the distinct binding distribution of CTCF.

When SNP discovery is carried out using ChIP-seq data, it might be expected that the highest SNP density occurs around significant ChIP peaks which are identified on the basis of normalized read coverage. We therefore investigated the distance between identified SNPs and the corresponding ChIP peak positions. Most SNPs from CTCF ChIP-seq data were indeed localized around -200 to $+200 \mathrm{bp}$ from CTCF peak binding positions, but interestingly, the number of CTCF SNPs dropped at the very center of CTCF peaks (Figure 2B). Since CTCF is evolutionarily conserved and its binding sites are also believed to be well conserved [25], we tested whether CTCF binding peaks were more highly conserved than the surrounding 


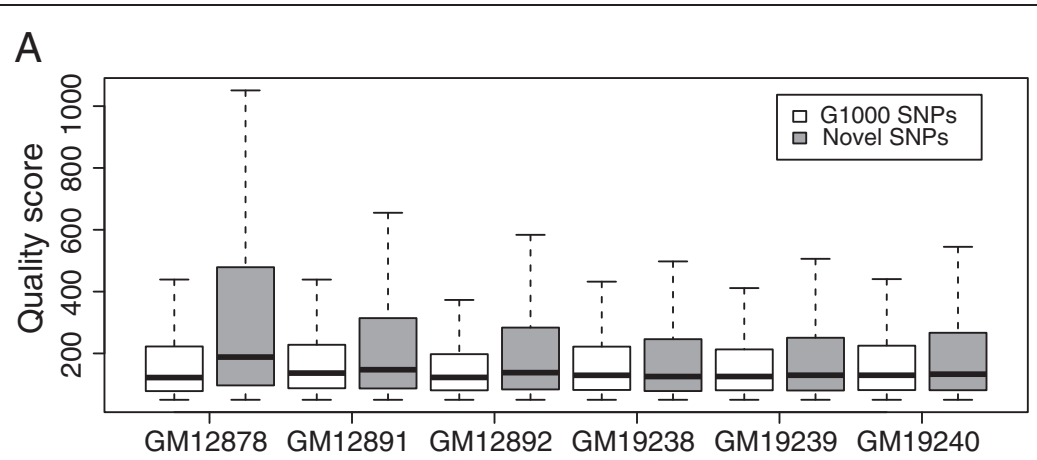

B

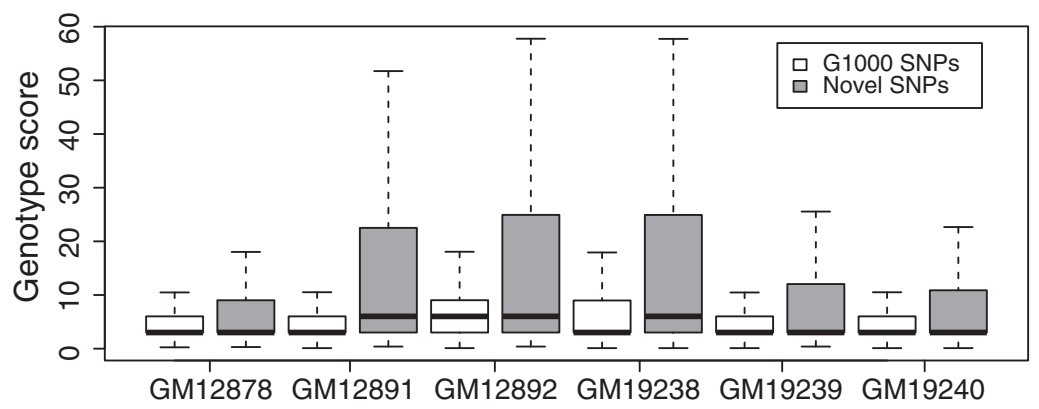

C
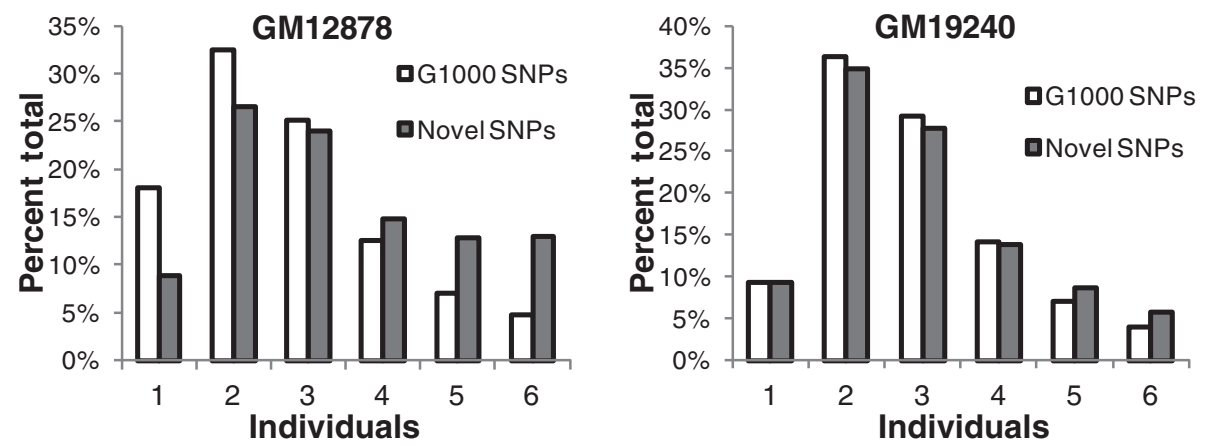

Figure 1 G1000 SNPs and novel SNPs are qualitatively similar. (A) SNP quality scores and (B) genotype quality scores of the G1000 SNPS and novel SNPs are shown for each of the indicated cell lines as standard box-plots. G1000 SNPs are those identified de novo from ChIP-seq data but overlap with the 1000 Genomes Project Pilot 2 SNP calls. (C) Individual distribution of SNPs. G1000 SNPs and novel SNPs discovered in GM12878 and GM19240 CTCF samples were categorized according to their individual distribution. For example, '1' represents SNPs found in only one of the six individuals, '2' represents SNPs found in two individuals, etc.

regions. When phastCons conservation scores from 28 vertebrate species [26] were profiled around CTCF peaks, a sharp increase in conservation was observed at CTCF binding centers (Figure 2C). The lower density of de novo SNPs that we observed at the centers of CTCF binding peaks is therefore likely due to increased evolutionary constraint at CTCF binding sites. We also observed a similar drop in SNP density of 1000 Genomes Project SNPs in at the center of CTCF binding sites (Additional file 1: Figure S6A).

In contrast to CTCF SNPs, SNPs discovered from RNAPII ChIP-seq data showed only a modest enrichment around RNAPII binding peaks (Figure 2D).
Phylogenetic conversation was higher over a broader range around RNAPII peak centers (Figure 2E) where the Pilot 2 SNP density was also lower (Additional file 1: Figure S6B). This lower overall SNP density around RNAPII binding sites as well as at TSS (Additional file 1: Figure S6C) may minimize any potential increase in the discovery of SNPs at RNAPII binding sites from RNAPII ChIP-seq data.

Analysis of the chromosomal distribution of SNPs discovered from CTCF ChIP-seq data showed that the relative SNP density on the $\mathrm{X}$ chromosome was markedly lower than that of the autosomes (Figure 3A). This was not a peculiarity of our SNP 


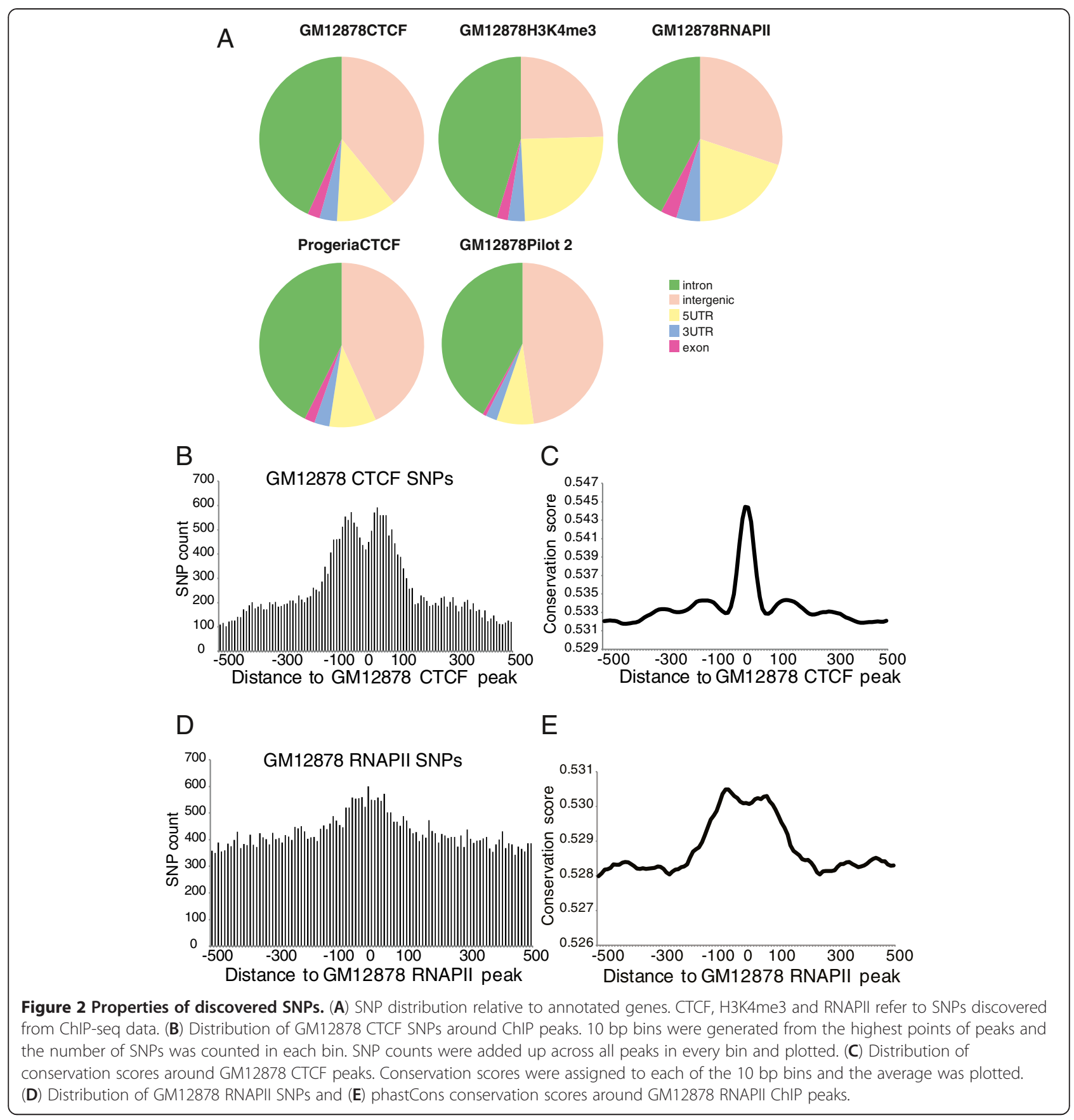

discovery pipeline as applied to ChIP-seq data, because when we analyzed Pilot 2 SNPs, we saw that the X chromosome similarly had a lower SNP density than autosomes (Figure 3B). The more pronounced bias for the $\mathrm{X}$ chromosome within SNPs discovered from ChIP-seq data (compare Figures $3 \mathrm{~A}$ and $3 \mathrm{~B}$ ) is not a SNP calling artifact because when we calculated the density of Pilot 2 SNPs within CTCF binding sites, the relative $\mathrm{X}$ chromosome SNP density was likewise lower than that of autosomes (Figure $3 \mathrm{C}$ ).
SNP discovery from ChIP-seq data is proportional to sequencing depth

From SNP discovery with CTCF ChIP-seq data in multiple cell lines, we found that the number of SNPs discovered varied considerably between cell lines (Table 1). Because the sequencing depth, that is, the total amount of sequence generated, differed between cell lines, we asked whether the difference in sequencing depths contributes to the difference in number of SNPs discovered. We found that the number of discovered SNPs indeed 

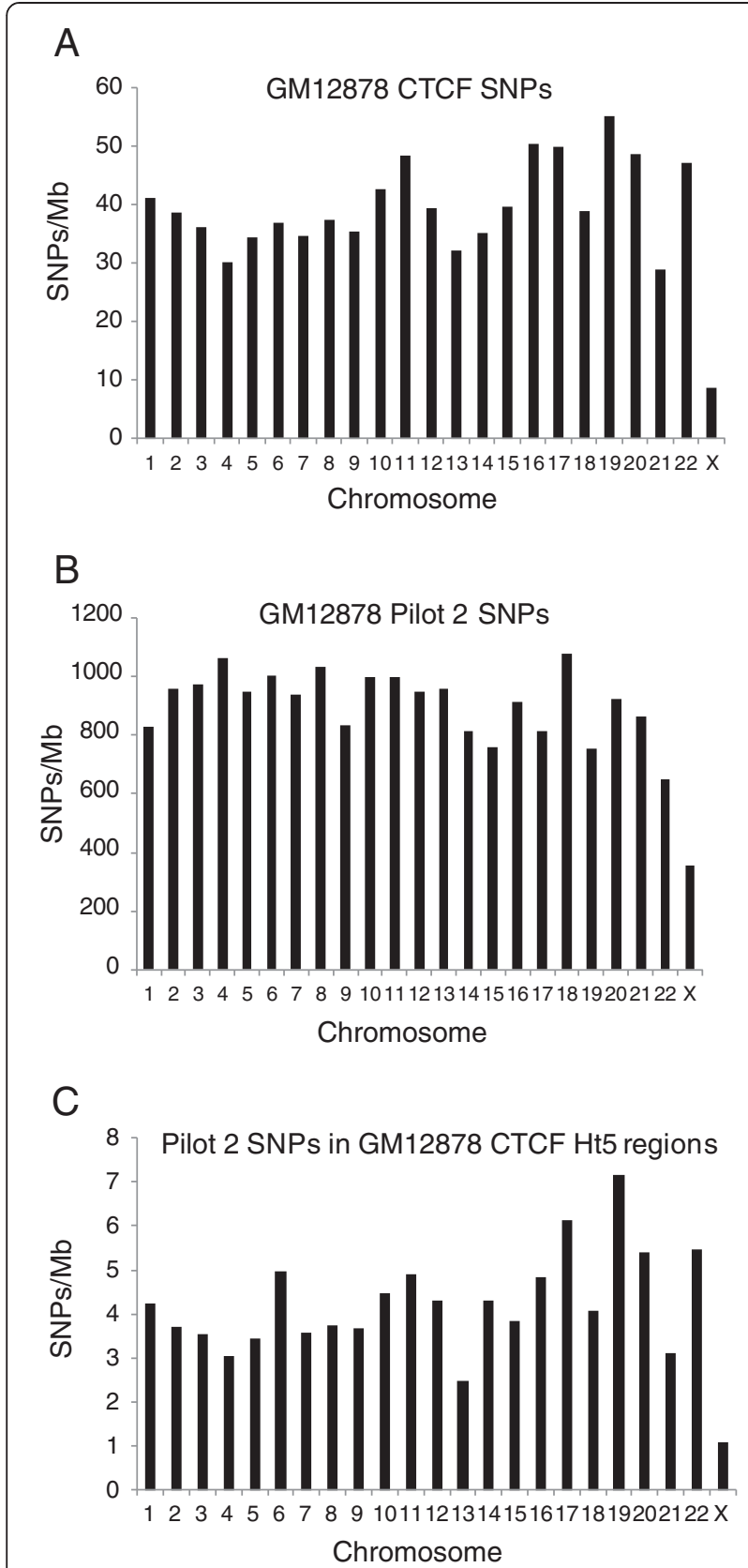

Figure 3 Chromosomal distribution of SNPs. Chromosomal distribution of SNPs is plotted as SNPs/Mb. Chromosome number is shown on the $X$ axis. Data is shown for (A) GM12878 CTCF SNPs (B) GM12878 Pilot 2 SNPs and (C) Pilot 2 SNPs within GM12878 CTCF ChIP Ht5 regions, which are regions with at least $5 \mathrm{X}$ coverage in ChIP-seq data.

correlated well with the number of aligned reads (Figure 4A).

Since SNPs within transcription factor binding regions are of the greatest interest from the perspective of understanding how genetic variation affects transcription factor binding, we were interested in understanding how

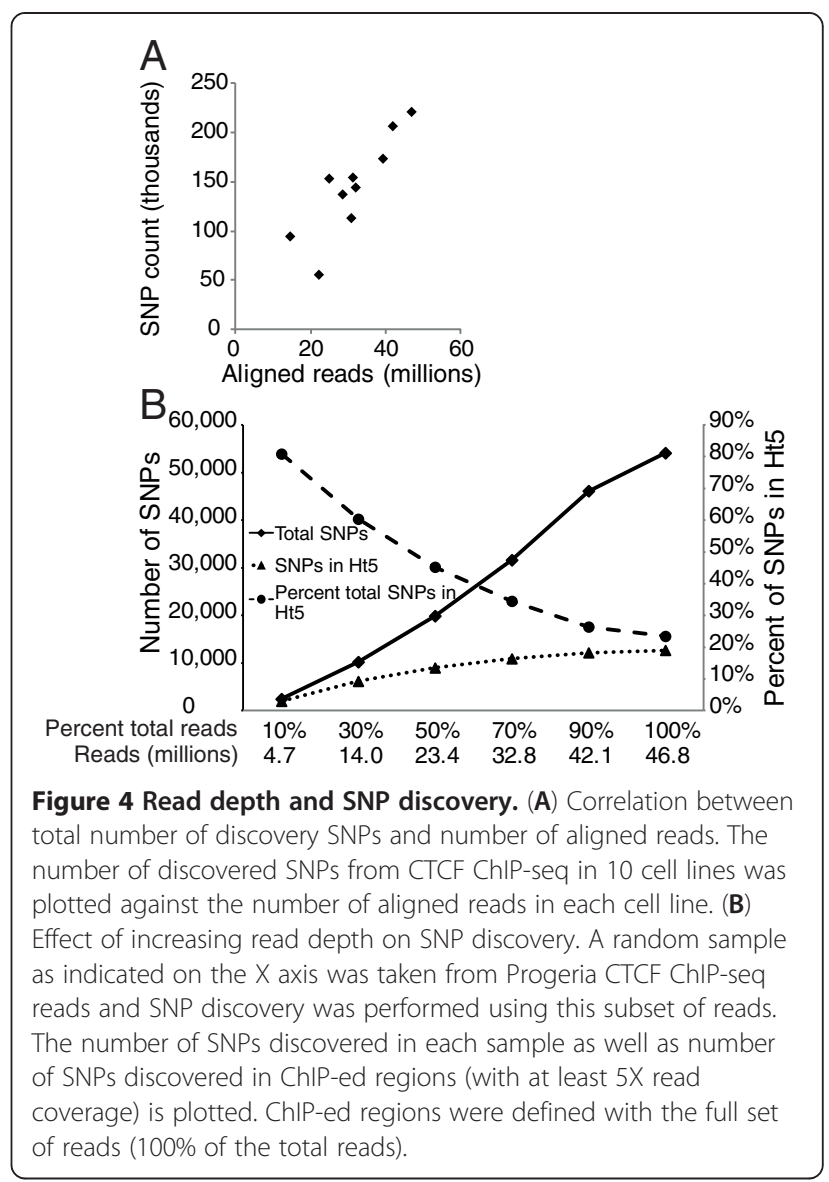

increased sequencing depth affected SNP discovery in those ChIP-ed regions. For this purpose, we used CTCF ChIP-seq data in Progeria fibroblasts where we had the greatest sequencing depth. We defined ChIP-ed regions as regions covered by 5 or more reads and analyzed the total number of SNPs, and SNPs discovered within ChIP-ed regions as a function of sequencing depth. While the total number of discovered SNPs increased linearly with increasing sequencing depth, the number of SNPs within ChIP-ed regions increased more slowly and almost plateaued after $\sim 32$ million reads. Furthermore, with increased sequencing depth, the percent of total SNPs within ChIP-ed regions decreased, suggesting that the additional SNPs discovered with deeper sequencing occur outside of the ChIP-ed regions and are therefore less likely to be relevant for transcription factor binding (Figure 4B).

\section{Consistent allele-specific binding bias at SNPs discovered from ChIP-seq data}

We have previously found that at a subset of assayable transcription factor binding sites, CTCF displays an allelic binding bias in that there is significant difference in its occupancy of the two alleles [16]. This allele-specific 
binding was consistent across individuals and appeared to be heritable. Our ability to accurately identify SNPs de novo from ChIP-seq data in principle enables us to detect allele-specific transcription factor binding bias even in the absence of prior genotype information. To confirm this, we first performed allele-specific binding bias analysis at SNP sites discovered de novo from CTCF ChIPseq data in the six lymphoblastoid cell lines (Methods).

A pairwise comparison of binding bias between two individuals, at shared heterozygous SNPs where the bias was statistically significant in at least one of the two individuals, showed that binding biases were largely concordant across individuals (Figure 5). The Spearman correlation coefficients of the binding biases between individuals were positive and highly significant in every case (Figure 5 inset tables). In particular, the bias values for most SNPs were located in the upper right or lower left quadrants, indicating that the direction of the binding bias was consistent between individuals. These results with SNPs we discovered de novo from ChIP-seq data recapitulate the findings from previous analysis which used then available genotypes released by the 1000 Genomes Project, and were mirrored in a similar analysis we performed using a more recent set of genotyped SNPs from the 1000 Genomes Project Pilot 2 release (Additional file 1: Figure S7). The set of significantly biased de novo discovered SNPs included in Figure 5 and the set of significantly biased Pilot 2 SNPs also overlap well (Additional file 1: Table S4).

We then extended this bias analysis to normal and disease fibroblasts that had not been previously genotyped, and found CTCF allelic binding bias between these two lines was similarly well correlated (Additional file 1: Figure S8). Thus, de novo SNP discovery using ChIP-seq data allows the measurement of allele-specific binding in the absence of any genotyping information and confirms the genetic basis of such allele-specific phenomena in primary and disease cells.

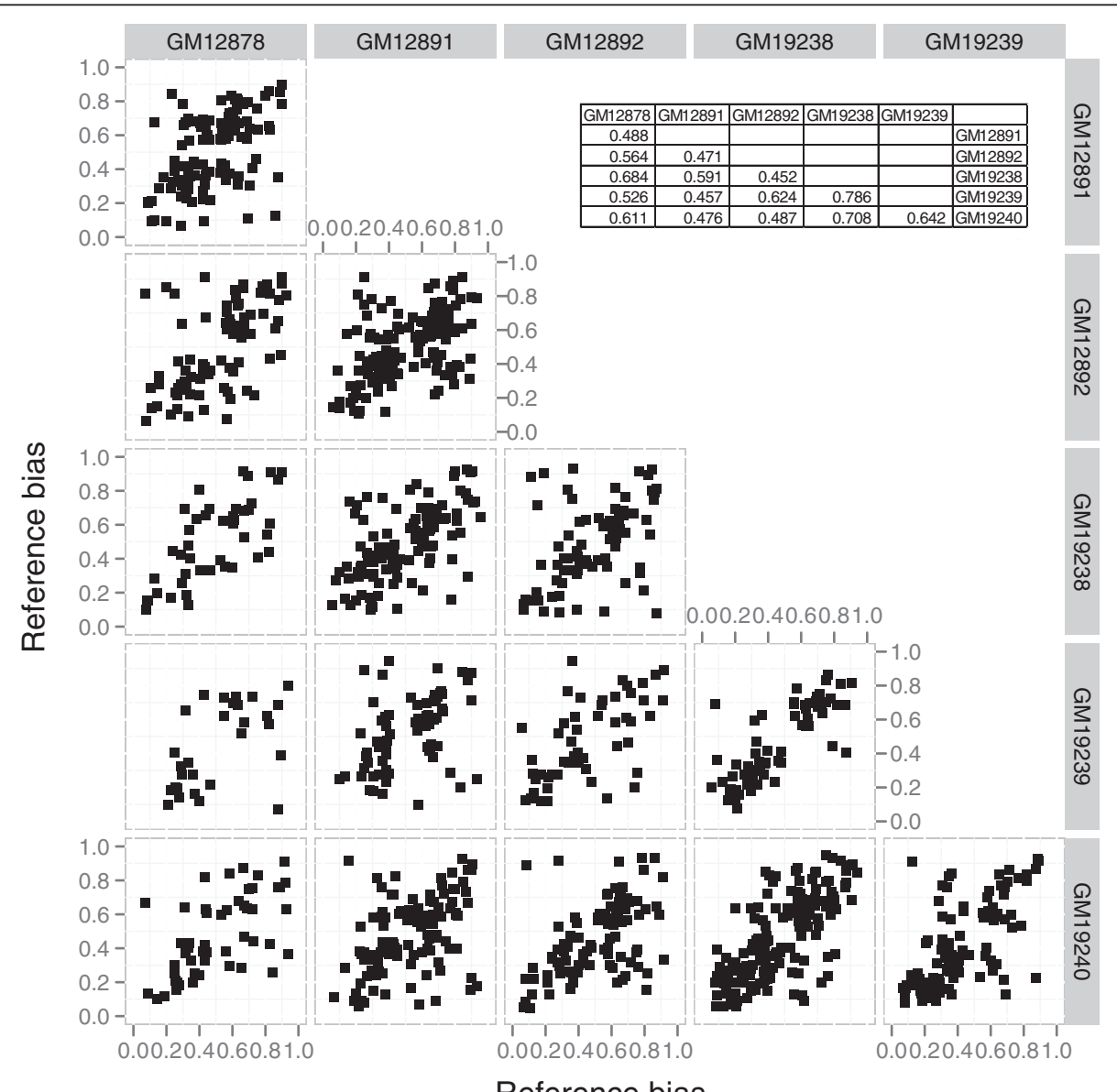

Figure 5 Bias analysis with discovery SNPs. Each plot is a scatter plot of percent reference allele (reads with reference allele/total reads) at shared heterozygous SNPs between two individuals as indicated on the top and right. SNPs that were significantly biased in at least one of the two individuals with an FDR corrected P-value less than 0.05 are included. The inset tables show the Spearman correlation of the bias between two individuals (top) and the Spearman P-values (bottom). 


\section{SNPs found in ChIP-seq data are informative for non- coding disease-associated variants}

To assess the biological utility of SNPs discovered directly from ChIP-seq data, we examined their overlap with SNPs that have been associated with human diseases through GWA studies [1]. Only 8 out of every 10,000 SNPs that were identified in the 1000 Genomes Pilot 2 Project were associated with a disease, but this proportion more than doubled when we looked at the ChIP-seq SNPs we identified. 17 out of every 10,000 SNPs that we found de novo by ChIP-seq overlapped with a disease associated SNP (Figure 6A). By restricting this overlap analysis to those de novo ChIP-seq SNPs that were also found in Pilot 2, we determined that this enrichment for disease SNPs within the ChIP-seq dataset is highly significant $(\mathrm{P}=8.2 \times 10-99$ by hypergeometric probability distribution). We found that a similarly higher proportion of ChIP-seq SNPs that we discovered across other cell lines that had not been genotyped by the 1000 Genomes project overlapped with disease-associated SNPs (Figure 6A).
Allele-specific binding biases could potentially reveal differences between normal and disease risk alleles, especially at non-coding SNPs. We therefore analyzed if any of the significantly biased allele-specific binding sites that we identified after genotyping and allele-specific analysis occurred near disease-associated SNPs identified by GWAS. We identified dozens of cases of significant allele-specific binding at distances ranging from $500 \mathrm{bp}$ to $5 \mathrm{~kb}$ from SNPs associated with various diseases (Figure 6B, Additional file 1: Table S5). Since this size range is well below the median haplotype block size of about $45 \mathrm{~kb}$ in humans $[3,27,28]$, a majority of these allele-specific binding sites we have identified are likely to occur on the same haplotype block as the GWAS SNP. An example of such a SNP is shown in Figure 6 C. In cells (GM19238 and GM19240) where the loci are heterozygous, the A allele is preferentially bound by CTCF. Consistent with this, in cells that have two $\mathrm{G}$ alleles at this location (GM19239, FB8460 and Progeria), CTCF binding is weaker than at the

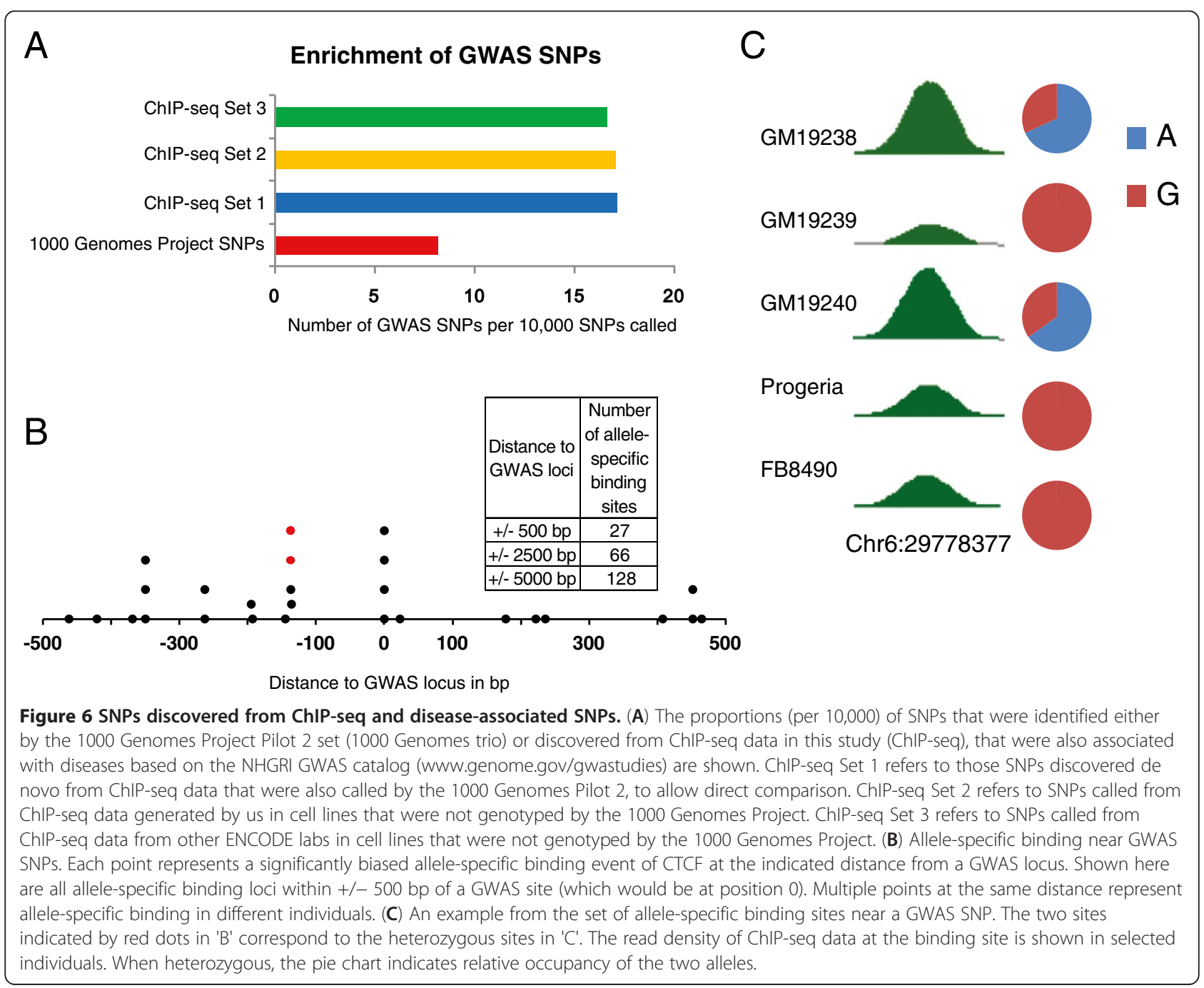


heterozygous sites. When they occur on the appropriate haplotypes, such allele-specific binding in non-coding regions could effectively represent a functional distinction between the nearby normal and risk alleles.

\section{Discussion}

SNP discovery and transcription factor binding allelic bias analysis from ChIPseq data

We developed an approach to simultaneously discover SNPs and perform transcription factor binding bias analysis using ChIP-seq data. Over 90\% of our discovered SNPs in six human lymphoblastoid cell lines overlap with the SNPs identified by the 1000 Genomes Project (Table 1). A similar proportion of SNPs that we discovered but were missed by the 1000 Genomes Project were in fact accurate as checked by Sanger sequencing. From the point of view of using such discovered SNPs to assess allele-specific binding events, the small number of cases where a heterozygous site is misidentified as homozygous do not constitute a problem since they are effectively "false negative" sites which would not be assayable for allele-specificity. Most discovered SNPs were close to transcription factor binding sites and the distribution of SNPs mimicked that of ChIP peaks (Figure 2). Due to the selectivity of ChIP, only a small fraction of all known SNPs were discovered; however, the majority of SNPs within ChIP-ed regions and therefore at transcription factor binding sites were recovered (Table 1). Furthermore, although increasing read depth leads to more SNPs, most SNPs in ChIP-ed regions were discovered with 20-30 million reads, which is a typical ChIP-seq dataset, suggesting that this type of analysis does not require very deep sequencing (Figure $4 \mathrm{~B}$ ).

The difference in SNP density on the X chromosome relative to autosomes is interesting. Many factors can lead to a difference in diversity between the $\mathrm{X}$ chromosome and autosomes, such as different effective population size, male mutation bias and natural selection $[29,30]$. We speculate that variants in functional regions on the X chromosome, for example, at transcription factor binding sites, are subject to a stronger natural selection bias than similar mutations on autosomes owing to the hemizygosity of the $\mathrm{X}$ chromosome. The difference in SNP density between the X chromosome and autosomes is therefore larger in SNPs discovered from CTCF ChIP-seq data, which are focused on functional regions, than in 1000 Genomes Project SNPs, which are largely intergenic.

\section{Utility of SNP genotyping from ChIP-seq and related data}

Since many disease-associated polymorphisms are in noncoding regions and are believed to affect gene regulation, a subset of such variants could affect transcription factor binding, chromatin structure or RNA processing. Determining the allelic effects of disease-associated SNPs on transcription factor binding, histone modifications, DNA methylation or DNaseI hypersensitivity would therefore be a powerful approach to directly assess the functional regulatory impact of non-coding polymorphisms. While it is of greatest interest to perform such studies on patient samples, one limitation is that complete genotypes are rarely available for primary patient samples. Our approach overcomes this limitation by enabling genotyping (SNP calling) and assessment of allele-specific effects directly from the ChIP or other functional experimental data, without requiring a separate genotyping dataset. We found that SNPs at transcription factor binding sites discovered from ChIP-seq data show an overrepresentation of disease-associated SNPs. Interestingly, previous studies have also shown an enrichment of GWAS SNPs in active intergenic chromatin regions that are defined partly by high CTCF binding [31]. Additionally, we found that many allele-specific binding events occur near non-coding disease-associated variants identified by GWAS. Such allele-specific binding potentially represents a functional distinction between the normal and disease alleles, which is currently not known for the majority of non-coding disease variants identified by GWAS. We readily identified the examples shown in Figure 6 and Additional file 1: Table S5 by analysis of a small number of ChIP-seq datasets. Similar analysis as we describe here, applied to the systematic datasets of genome-wide transcription factor binding, chromatin status, and gene expression will undoubtedly identify such distinctions for many more disease-associated loci, which can then be tested in patient samples.

While genotyping using arrays or deep sequencing might appear to be straightforward, arrays generally cover only a small subset of all possible polymorphisms, and whole genome resequencing requires far deeper sequencing coverage than required for typical ChIP-seq or RNA-seq assays. The fact that we could identify and verify SNPs that were missed in the 1000 Genomes Project genotypes suggests that the selective focus of our method on biochemically functional elements offers an advantage even over whole-genome sequencing, likely because our sequencing coverage over these loci is higher at these enriched loci. While it is possible that a subset of phenotypic effects are due to rare "private" variants, distinguishing whether a rare non-coding variant has a functional consequence or is neutral is challenging. The approach outlined here can not only identify such rare variants but also immediately establish whether it significantly affects the experimental assay that was used to identify it in the first place. We propose that by analogy to exome sequencing which is aimed at identifying variants in coding regions, the approach we have used here can be considered to be "targetome" sequencing, in which variants in functional non-coding regions are identified along with information on how these variants 
affect enrichment in the functional assay. Despite its potential, technical issues are likely to arise in certain domains. For example, one application might be to evaluate the allelic effects of non-coding somatic mutations and germline polymorphisms in cancer, a disease where the underlying affected cells are readily identifiable (as tumors) and can be isolated for experimental analysis. However, cancer cells are often aneuploid and tumor samples from patients are often genetically heterogeneous, so bias analysis for transcription factor binding or chromatin must account for the background allele composition. SNP arrays have been used to determine copy number differences in cancer cells [32], so in parallel to ChIP-seq analysis as outlined here, it should be possible to determine the copy number of each allele in the input genomic DNA from the same sample, and use this quantitative information as the background while calculating the significance of any allele-specific binding bias.

Although our study focused on ChIP-seq data, combining ChIP-seq and RNA-seq allele-specific analysis in the same samples will clarify the relationship between non-coding regulatory polymorphisms and allele-specific gene expression. Since transcription factor and chromatin changes are likely some distance away from the transcribed region along the linear chromosome, such an understanding will also require knowledge of SNP phasing along individual chromosomes, but haplotype inference based on statistical approaches or direct haplotype resolved sequencing methods can fill this gap $[33,34]$. Allele-specific events of all types can thus be measured in primary and disease cells and will shed light on how genotypic variation corresponds to variation in phenotype, including diseases.

\section{Conclusions}

Our approach demonstrates that it is possible to discover SNPs with very high accuracy and simultaneously perform transcription factor binding bias analysis directly from ChIP-seq data. While the overwhelming majority of SNPs discovered from ChIP-seq data coincide with published genotype data, the small number of novel SNPs that we found appear to be indistinguishable from previously published variants based on several criteria. Using SNPs discovered de novo from ChIP-seq data, we confirmed and extended previous observations that allele-specific biases tend to occur towards the same allele across individuals and are therefore consistent with an underlying genetic basis for these binding differences. SNPs discovered from ChIP-seq data are strongly enriched for disease-associated variants, and allelespecific binding at many of these SNPs could allow a functional distinction to be made between normal and disease haplotypes.

\section{Methods}

\section{Data source}

All CTCF and RNAPII ChIP-seq and peak finding was performed by our group as described earlier [18] and the data is available from the ENCODE project web site (http://genome.ucsc.edu/ENCODE/). H3K4me3 and H3K27me3 ChIP-seq data was also downloaded from the ENCODE website. RNA-seq data for GM12891 was from a previously published study [35]. The statistics of these data sets are in Table 1 and Additional file 1: Table S6. 1000 Genome Project SNP calls were as in their Pilot 2 release [4]. The NHGRI GWAS catalog (www.genome. gov/gwastudies) was downloaded and the data as accessed on Feb 232012 was used for our analysis.

\section{SNP calling with GATK}

Raw ChIP-seq sequencing reads were aligned to the human reference genome NCBI36 (hg18) with BWA [21]. To ensure optimal alignment, we used the reference genome provided with GATK that contained all 24 chromosome sequences as well as additional contigs that have not been assembled. GATK was then used to mark duplicated reads, realign around insertions/deletions and recalibrate quality scores for the alignments. The Unified Genotyper was used to generate initial SNP calls in vcf format which was then further filtered. The initial SNP calls were evaluated with a Gaussian Mixture Model (GATK Variant Recalibrator) and outliers were discarded. After this initial filtering, the SNPs in the following categories were then filtered out: more than 2 SNPs within 10 base pairs, more than $10 \%$ overlapping reads are not uniquely mapped, SNPs with quality scores less than 50, SNPs overlapping with repeat regions [22], SNPs within 5 bp of insertions/deletions, SNPs located within regions with exceptionally high read coverage [23].

\section{Allelic binding bias analysis}

Bias analysis was performed as described previously [16]. To avoid alignment biases favoring reads containing the reference allele, we constructed individual-specific reference genomes that contained the SNPs discovered in that individual from the ChIP-seq data, representing both the reference and the alternate allele in the case of heterozygous SNPs. We then carried out a second round of alignments to these individual-specific genomes. We counted the number of reads covering each allele at heterozygous SNPs and expressed the ChIP-seq binding bias as the proportion of reads that contain the reference allele. We calculated the significance of this bias using a binomial model, assuming under the null hypothesis that both alleles of a heterozygous SNP would be equally represented in the ChIP DNA. $\mathrm{P}$-values were corrected for multiple testing using the false discovery rate (FDR) on the set of SNPs where the difference of allele counts between the two alleles was at least 6 . The lists of significantly biased binding sites from de novo 
identified SNPs as well as from 1000 Genomes Project Pilot 2 SNPs are provided as Additional file 2: Table S7 and Additional file 3: Table S8 respectively.

\section{Additional files}

Additional file 1: Figure S1. Diagram of SNP discovery pipeline. Figure S2. Numbers of Pilot 2 SNPs rediscovered correlate with ChIP-seq coverage. For each trio cell line, the percentage of Pilot 2 SNPs rediscovered with ChIP-seq data is plotted together with percent of the genome with at least 5X coverage from ChIP-seq. Figure S3. Validation of de novo discovered SNPs by genomic sequencing. The top row shows examples of SNPS discovered de novo from ChIP-seq data that were also genotyped in that individual by the 1000 Genomes Pilot 2 Project. The remainder are examples of SNPs discovered de novo from ChIP-seq data but missed in the 1000 Genomes Pilot 2 set in that individual (GM cell lines) or found in ungenotyped lines (HUVEC, Progeria). The top of each panel shows the genomic DNA sequence, with the SNP at the center in bold. Chromosomal coordinates, transcription factor/histone modification, and cell line are listed below the chromatogram. Figure S4. SNP calling in low coverage regions. (A) Location overlap and genotype overlap between CTCF ChIP-seq SNPS and Pilot 2 SNPs. Location overlap is when the SNP location and alleles match, but sometimes only one allele of a heterozygous genotype is observed in the other set. Genotype overlap refers to an exact genotype match. (B) Percent heterozygosity for CTCF ChIP-seq discovery SNPs and Pilot 2 SNPs. (C) Read number filtering increases discovery SNP heterozygosity and genotype overlap with Pilot 2 SNPs. SNPs covered by less than the indicated number of reads were filtered out. Blue bars represent the number of SNPs passing the filter. Red squares represent SNP heterozygosity and green triangles represent the percent genotype overlap with Pilot 2 SNPs, both on the secondary Y axis on the right. Figure S5. Individual distribution of SNPs. G1000 SNPs and novel SNPs discovered in the indicated GM cell lines. CTCF ChIP-seq samples were categorized according to their individual distribution. '1' represents SNPs found in only one of the six individuals, '2' represents SNPs found in two people and so on. Figure S6. Pilot 2 SNP distribution around (A) CTCF and (B) RNAPII ChIP peak centers and (C) transcription start sites. (D) Conservation scores around transcription start sites. All distances are in bp. Figure S7. CTCF allelic binding bias at Pilot 2 SNPs was plotted similarly as in Fig. 5. The inset tables show the Spearman correlation coefficients (top) and Spearman $P$ values (bottom). Figure S8. CTCF allelic binding bias at discovered SNPs in Progeria and FB8470 (normal) fibroblast cells. Table S1. Description of apparent errors. This table lists all 6 discrepancies that we observed between genotypes called from ChIP-seq data and our genomic Sanger sequencing validation (127 out of 133 were exactly correct). For errors 1 and 3, the ChIP-seq data recovered the alternate allele and called it homozygous, but the reference allele was apparently not observed at sufficient coverage. Errors 2 and 4 are discrepant between the ChIP-seq and Sanger genotyping, but our ChIP-seq call matched the 1000 Genomes Pilot 2 genotype. For errors 5 and 6, the ChIP-seq data called it heterozygous and Sanger sequencing reported homozygous (similar to errors 2and 4), but the two alleles reported by ChIP-seq correspond to the two alleles known to occur at that position (in other individuals) according to dbSNP 129. Table S2. Indels called from ChIP-seq data overlap with 1000 Genomes Project indel calls. Table S3. Novel SNPs found by ChIP-seq overlap with SNPs found in other individuals in the same population in the 1000 Genomes Project low coverage data. Table S4. Overlap between biased (that is, allele-specific) SNPs discovered from ChIP-seq data and biased Pilot 2 SNPs. Table S5. Significantly biased allele-specific CTCF binding sites within 500 bp of a GWAS SNP locus. $P$-val refers to the significance of the allele-specificity binding bias at a heterozygous SNP. Table S6. SNP calling from H3K4me3 and/or H3K27me3 ChIP-seq data in 17 additional cell lines (ENCODE data) as well as from RNA-seq data in GM12891 (from Toung et al., Genome Res. (2011) 21:991-8).

Additional file 2: $C T C F$ allele-specific binding at SNPs discovered de novo from CTCF ChIP-seq. SNPs with an FDR corrected bias $P$ value of less than 0.05 are included. Each tab contains information for one individual.

Additional file 3: CTCF allele-specific binding at Pilot 2 SNPs. SNPs with an FDR corrected bias $P$ value of less than 0.05 are included. Each tab contains information for one individual.

\section{Competing interests}

The authors declare no competing interests.

\section{Acknowledgments}

We thank B.K. Lee, L. Song, G. Crawford and our ENCODE Project collaborators for assistance with data collection, and the Progeria Research Foundation for cell lines. This work was supported by an NIH/NHGRI ENCODE Consortium grant U54 HG004563 and by R01 CA130075.

\section{Authors' contributions}

$\mathrm{VRI}$ and $\mathrm{YN}$ conceived of and designed the study. YN and AB carried out the computational analysis. AWH performed the experimental validation. VRI and YN wrote the manuscript. All authors read and approved the final manuscript.

Received: 24 May 2012 Accepted: 5 September 2012

Published: 5 September 2012

\section{References}

1. Hindorff LA, Sethupathy P, Junkins HA, Ramos EM, Mehta JP, Collins FS, Manolio TA: Potential etiologic and functional implications of genome-wide association loci for human diseases and traits. Proc Natl Acad Sci U S A 2009, 106:9362-9367.

2. Hirschhorn JN, Gajdos ZK: Genome-wide association studies: results from the first few years and potential implications for clinical medicine. Annu Rev Med 2011, 62:11-24.

3. The International HapMap Consortium: A second generation human haplotype map of over 3.1 million SNPs. Nature 2007, 449:851-861.

4. The 1000 Genomes Project Consortium: A map of human genome variation from population-scale sequencing. Nature 2010, 467:1061-1073.

5. Manolio TA, Collins FS, Cox NJ, Goldstein DB, Hindorff LA, Hunter DJ, Carthy MI, Ramos EM, Cardon LR, Chakravarti A, et al: Finding the missing heritability of complex diseases. Nature 2009, 461:747-753.

6. Dickson SP, Wang K, Krantz I, Hakonarson H, Goldstein DB: Rare variants create synthetic genome-wide associations. PLOS Biol 2010, 8:e1000294. 7.

7. McClellan J, King M, McClellan J, King MC: Genetic heterogeneity in human disease. Cell 2010, 141:210-217.

8. Wang K, Dickson SP, Stolle CA, Krantz ID, Goldstein DB, Hakonarson H: Interpretation of association signals and identification of causal variants from genome-wide association studies. Am J Hum Genet 2010, 86:730-742.

9. Birney E, Lieb JD, Furey TS, Crawford GE, Iyer VR: Allele-specific and heritable chromatin signatures in humans. Hum Mol Genet 2010, 19:R204-R209.

10. Cheung VG, Spielman RS: Genetics of human gene expression: mapping DNA variants that influence gene expression. Nat Rev Genet 2009, 10:595-604.

11. Seibold MA, Wise AL, Speer MC, Steele MP, Brown KK, Loyd JE, Fingerlin TE, Zhang W, Gudmundsson G, Groshong SD, et al: A common MUC5B promoter polymorphism and pulmonary fibrosis. N Engl J Med 2011, 364:1503-1512.

12. Meyer KB, Maia AT, O'Reilly M, Teschendorff AE, Chin SF, Caldas C, Ponde BA: Allele-specific up-regulation of FGFR2 increases susceptibility to breast cancer. PLoS Biol 2008, 6:e108.

13. Meyer KB, Maia AT, O'Reilly M, Ghoussaini M, Prathalingam R, Porter-Gill P, Ambs S, Prokunina-Olsson L, Carroll J, Ponder BA: A functional variant at a prostate cancer predisposition locus at 8q24 is associated with PVT1 expression. PLoS Genet 2011, 7:e1002165.

14. Bond GL, Hu W, Bond EE, Robins H, Lutzker SG, Arva NC, Bargonetti J, Bartel $F$, Taubert $H$, Wuerl P, et al: A single nucleotide polymorphism in the MDM2 promoter attenuates the p53 tumor suppressor pathway and accelerates tumor formation in humans. Cell 2004, 119:591-602.

15. Knappskog S, Bjornslett M, Myklebust LM, Huijts PE, Vreeswijk MP, Edvardsen H, Guo Y, Zhang X, Yang M, Ylisaukko-Oja SK, et al: The MDM2 promoter SNP285C/ $309 \mathrm{G}$ haplotype diminishes Sp1 transcription factor binding and reduces risk for breast and ovarian cancer in Caucasians. Cancer Cell 2011, 19:273-282.

16. McDaniell R, Lee BK, Song L, Liu Z, Boyle AP, Erdos MR, Scott L, Morken MA, Kucera KS, Battenhouse A, et al: Heritable individual-specific and allelespecific chromatin signatures in humans. Science 2010, 328:235-239.

17. Kasowski M, Grubert F, Heffelfinger C, Hariharan M, Asabere A, Waszak SM, Habegger L, Rozowsky J, Shi M, Urban AE, et al: Variation in transcription factor binding among humans. Science 2010, 328:232-235.

18. Lee BK, Bhinge AA, Battenhouse A, McDaniell RM, Liu Z, Song L, Ni Y, Birney E, Lieb JD, Furey TS, et al: Cell-type specific and combinatorial usage of diverse transcription factors revealed by genome-wide binding studies in multiple human cells. Genome Res 2012, 22:9-24. 
19. DePristo MA, Banks E, Poplin R, Garimella KV, Maguire JR, Hartl C, Philippakis AA, del Angel G, Rivas MA, Hanna M, et al: A framework for variation discovery and genotyping using next-generation DNA sequencing data. Nat Genet 2011, 43:491-498.

20. McKenna A, Hanna M, Banks E, Sivachenko A, Cibulskis K, Kernytsky A, Garimella K, Altshuler D, Gabriel S, Daly M, DePristo MA: The genome analysis toolkit: a map reduce framework for analyzing next-generation DNA sequencing data. Genome Res 2010, 20:1297-1303.

21. Li H, Durbin R: Fast and accurate long-read alignment with BurrowsWheeler transform. Bioinformatics 2010, 26:589-595.

22. Benson G: Tandem repeats finder: a program to analyze DNA sequences. Nucleic Acids Res 1999, 27:573-580.

23. Pickrell JK, Gaffney DJ, Gilad Y, Pritchard JK: False positive peaks in Ch IP seq and other sequencing-based functional assays caused by unannotated high copy number regions. Bioinformatics 2011, 27:2144-2146.

24. Ebersberger I, Metzler D, Schwarz C, Paabo S: Genomewide comparison of DNA sequences between humans and chimpanzees. Am J Hum Genet 2002, 70:1490-1497.

25. Phillips JE, Corces VG: CTCF: master weaver of the genome. Cell 2009, 137:1194-1211.

26. Miller W, Rosenbloom K, Hardison RC, Hou M, Taylor J, Raney B, Burhans R, King DC, Baertsch R, Blankenberg D, et al: 28-way vertebrate alignment and conservation track in the UCSC Genome Browser. Genome Res 2007, 17:1797-1808

27. Guryev V, Smits BM, van de Belt J, Verheul M, Hubner N, Cuppen E: Haplotypeblock structure is conserved across mammals. PLoS Genet 2006, 2:e121.

28. The International HapMap Consortium: A haplo type map of the human genome. Nature 2005, 437:1299-1320.

29. Ellegren $\mathrm{H}$ : The different levels of genetic diversity in sex chromosomesand autosomes. Trends Genet 2009, 25:278-284.

30. Gottipati S, Arbiza L, Siepel A, Clark AG, Keinan A: Analyses of X-linked andautosomal genetic variation in population-scale whole genome sequencing. Nat Genet 2011, 43:741-743.

31. Ernst J, Kellis M: Discovery and characterization of chromatin states for systematic annotation of the human genome. Nat Biotechnol 2010, 28:817-825

32. Attiyeh EF, Diskin SJ, Attiyeh MA, Mosse YP, Hou C, Jackson EM, Kim C, Glessner J, Hakonarson H, Biegel JA, Maris JM: Genomic copy number determination in cancer cells from single nucleotide polymorphism microarrays based on quantitative genotyping corrected for aneuploidy. Genome Res 2009, 19:276-283.

33. Fan HC, Wang J, Potanina A, Quake SR: Whole-genome molecular haplotyping of single cells. Nat Biotechnol 2011, 29:51-57.

34. Kitzman JO, Mackenzie AP, Adey A, Hiatt JB, Ng SB, Alkan C, Qiu R, Eichler $\mathrm{EE}$, Shendure J: Haplotype-resolved genomesequencing of a Gujarati Indian individual. Nat Biotechnol 2011, 29:59-63.

35. Toung JM, Morley M, Li M, Cheung VG: RNA-sequence analysis of human B-cells. Genome Res 2011, 21:991-998.

doi:10.1186/1471-2156-13-46

Cite this article as: Ni et al:: Simultaneous SNP identification and assessment of allele-specific bias from ChIP-seq data. BMC Genetics 2012 13:46.

\section{Submit your next manuscript to BioMed Central and take full advantage of:}

- Convenient online submission

- Thorough peer review

- No space constraints or color figure charges

- Immediate publication on acceptance

- Inclusion in PubMed, CAS, Scopus and Google Scholar

- Research which is freely available for redistribution 